many diseases once thought to be rheumatoid are recognised as separate entities. Among these are systemic lupus erythematosus and the seronegative spondarthroses (in particular, ankylosing spondylitis)-entities in which treatment with gold is at worst potentially harmful and at best usually unhelpful. The side effects ascribed to gold have also changed: jaundice has virtually disappeared since the introduction of disposable needles and syringes; proteinuria and the nephrotic syndrome are usually recognised to be due to secondary amyloidosis. Cutaneous manifestations remain a problem, but are now seen as a reason for temporary discontinuation rather than as an absolute contraindication to treatment. Bone marrow depression, occasionally fatal, still occurs; but clinicians are now more aware of the problem, and with careful monitoring ${ }^{3}$ the number of deaths reported annually to the Committee on Safety of Medicines has not paralleled the increased use of gold. ${ }^{4}$

Methods of prescribing gold are still changing. Once it was given as four injections each of $250 \mathrm{mg}$. In the Empire Rheumatism Council controlled trial the course was of 20 injections of $50 \mathrm{mg}$. In the period of surveillance after that trial many patients relapsed, and monthly maintenance injections were introduced. A fixed-dosage regimen of gold on this pattern was compared with flexible regimens of cytotoxic drugs and with penicillamine..$^{5}$ In the last few years ideas have changed again, and flexible regimens have been tailored to the patients' needs ${ }^{7}$ with improved results and less toxicity. When gold is used earlier in the course of the disease $^{8}$ remissions will occur sooner; and in these circumstances the frequency of injections should be reduced ${ }^{7}$ at the time of clinical remission to lessen the risk of toxicity.

At the end of 50 years the consensus is that gold is still useful in rheumatoid arthritis. The recent introduction of a new effective oral gold preparation may change our ideas again. ${ }^{9}$ And it is always possible that 1979 may see the discovery of a real cure for rheumatoid arthritis-or of its cause.

${ }^{1}$ Forestier, J M, Bulletin et Mémoires de la Société Médicale des Hôpitaux de Paris, 1929, 53, 323.

2 Research Subcommittee of the Empire Rheumatism Council, Annals of the Rheumatic Diseases, 1961, 20, 315.

${ }^{3}$ Kay, A G L, British Medical fournal, 1976, 1, 1266.

4 Gumpel, J M, British Medical fournal, 1978, 1, 215.

5 Woodland, J, et al, Annals of the Rheumatic Diseases, 1974, 33, 399.

${ }^{6}$ Huskisson, E C, et al, Annals of the Rheumatic Diseases, 1974, 33, 532.

7 Gumpel, J M, Rheumatology and Rehabilitation, 1976, 15, 217.

${ }^{8}$ Luukkainen, R, Kajander, A, and Isomäki, H, Scandinavian fournal of Rheumatology, 1977, 6, 189.

${ }^{9}$ Finkelstein, A E, et al, Annals of the Rheumatic Diseases, 1976, 35, 251.

\section{Preventing endocarditis}

Last year the American Heart Association made revised recommendations for antibiotic cover of procedures known to cause bacteraemia. ${ }^{1}$ These include not only dental extraction and other surgery in the mouth and throat but various operations on the urinary and lower alimentary tracts. Another procedure-namely, transrectal biopsy of the prostate-has recently joined the list. ${ }^{2}$ The new recommendations evidently owed much to the experimental studies of D T Durack, a member of the committee formulating them, who argued from his results that only a large and preferably parenteral dose of a bactericidal antibiotic or combination of antibiotics is adequate. The regimen proposed for dental operations is a large parenteral dose of penicillin followed by eight doses of $0.5 \mathrm{~g}$ penicillin $\mathrm{V}$ at 6-hour intervals or, in patients allergic to penicillin, intravenous vancomycin followed by a similar course of erythromycin. To guard against infection by the more resistant enterococcus in abdominal operations, the proposed cover is even more formidable-penicillin or ampicillin plus gentamicin or streptomycin given parenterally in three doses.

We commented that these proposals placed a formidable responsibility on those obeying them, and suggested that Durack's experimental model was too severe a test for his conclusions to apply literally to transient bacteraemia in man. ${ }^{3}$ It consisted of producing vegetations on the aortic valves of rabbits by inserting catheters into the cardiac chambers and inducing bacteraemia with a massive intravenous injection of streptococci. The criterion for adequate treatment was sterilising the blood in 24 hours. We questioned this model on the ground that, as bacteria on these vegetations may be less accessible than those on the intact endothelial surface of even a valve deformed by disease, this type of experiment is to some extent an exercise in treating rather than preventing endocarditis; and 24 hours may be too short a time in which to expect sterilisation.

This criticism of the method has now been echoed by Petersdorf ${ }^{4}$-even though he was co-author with Durack of the two principal accounts of these experiments. ${ }^{5} \mathrm{He}$ calls his paper "Antimicrobial prophylaxis of bacterial endocarditis: prudent caution or bacterial overkill ?" apparently having reacted against the "hyperaggressive recommendations" and particularly those of Kaye, ${ }^{7}$ who proposes even larger doses of the same parenteral antibiotics continued for three days. Petersdorf points out not only that patients dislike injections and that "intravenous therapy with a drug like vancomycin in the dentist's office seems totally impractical," but that Kaye's proposals call for three days in hospital and could cost as much as $\$ 1000$. His own proposals are much simpler. Parenteral prophylaxis against Streptococcus viridans endocarditis, with a single dose of procaine penicillin and streptomycin, "should be reserved for high-risk patients such as those with prosthetic heart valves, and should not be used for patients without such intracardiac devices." This alone among his suggestions is disputable: is not an existing rheumatic valvular defect or even a clear history of rheumatic fever another indication-not to mention a previous attack of bacterial endocarditis? For "most other patients" he suggests a $2 \mathrm{~g}$ oral dose of penicillin $\mathrm{V}$ followed by three doses of $0.5 \mathrm{~g}$ or similar treatment with erythromycin if the patient is allergic to penicillin-emphasising the need for only three rather than eight further doses. Parenteral treatment is needed, however-as he acknowledges - to prevent enterococcal infection, and he recommends penicillin or ampicillin plus gentamicin. The treatment could be completed in 12 to 24 hours.

The observations of Shanson and his colleagues, who made blood cultures from treated and control patients at the London Hospital two minutes after dental extraction, should encourage us to rely on a single large oral prophylactic dose of antibiotic. ${ }^{8}$ In the past such cultures have often proved little, because antibiotic in the culture could have prevented the growth of surviving bacteria. But Shanson added penicillinase to one of two bottles after four hours' incubation and to the other after 24 hours, so that any surviving bacteria could multiply. There were 40 patients in each of three groups. Aerobic streptococci grew from the blood of 14 controls and from five given penicillin $\mathrm{V}$ and two given amoxycillin (each in a dose of $2 \mathrm{~g}$ one hour before extraction). These growths were in bottles to which penicillinase had been added after four hours; when it 
was added after 24 hours all but one were sterile. The findings in cultures for anaerobes were similar. In a separate study Shanson showed that blood concentrations of amoxycillin were higher and much better sustained than those of penicillin V. This should surprise no one; not only is penicillin $\mathrm{V}$ incompletely absorbed, but much of what is absorbed is degraded to penicilloic acid, so that no more than a third of the dose circulates in active form. ${ }^{9}$ Amoxycillin suffers from no such defects, and may therefore be the better oral prophylactic, certainly deserving a place in any future trials.

Petersdorf's paper concludes with a plea that the medical and dental professions should keep much better records. No statistical proof exists of the efficacy of any of these procedures, and we agree that if we are to rely so much on oral antibiotics we should obtain such proof.

American Heart Association, Circulation, 1977, 56, 139A

2 Ashby, E C, Rees, M, and Dowding, C H, British Medical fournal, 1978, $2,1263$.

3 British Medical fournal, 1977, 2, 1564

4 Petersdorf, R G, American fournal of Medicine, 1978, 65, 22.

5 Durack, D T, and Petersdorf, R G, Fournal of Clinical Investigation, 1973, 52, 592.

'Pelletier, LL, jr, Durack, D T, and Petersdorf, R G, fournal of Clinical Investigation, 1975, 56, 319.

Kaye, D, Infective Endocarditis. Baltimore, University Park Press, 1976.

${ }^{8}$ Shanson, D C, Cannon, P, and Wilks, M, fournal of Antimicrobial Chemotherapy, 1978, 4, 431.

${ }^{9}$ Hellström, K, Rosen, A, and Swahn, $\AA$, Clinical Pharmacology and Therapeutics, 1974, 16, 826 .

\section{Polycystic disease of the kidneys}

Two main types of bilateral polycystic disease have been recognised: the infantile and the adult (the multiple renal cysts associated with certain syndromes of multiple congenital malformations and chromosomal abnormalities ${ }^{1}$ are very rare). The infantile type is usually fatal in infancy, often soon after birth; it also is associated with cystic malformations of the intrahepatic bile ducts, with apparently autosomal recessive inheritance, whereas the adult type is inherited as an autosomal dominant. But this now seems to be too simple a picture of the range of polycystic kidney disease.

Typically, the adult type presents in the fourth or fifth decade, and clinical features may include recurrent macroscopic haematuria; loin pain, due to infection or haemorrhage into cysts; clot colic; hypertension; and swelling of the abdomen due to the enlarging kidneys, which seem also to be more vulnerable to trauma from accidents. This type of the disease is associated with intracranial aneurysms and sometimes hyperuricaemia. Solitary or multiple cysts may also occur in the liver and occasionally in other organs such as the pancreas and spleen. ${ }^{2}$ The liver cysts do not result in cirrhosis or portal hypertension, but occasionally they become infected or painful because of haemorrhage into them. In the past patients usually died from complications associated with hypertension, subarachnoid haemorrhage after rupture of an intracranial aneurysm, or renal failure. The adult type of polycystic disease is an important cause of severe chronic renal failure, and is the primary underlying renal disease in about $8 \%$ (3978) of the 47741 adult patients in the European Dialysis and Transplantation Registry. ${ }^{3}$ It may, however, remain subclinical, being observed for the first time at necropsy in elderly patients dying from other causes. Now that even the badly affected patients often survive for a long time, thanks to maintenance haemodialysis and renal transplantation, adequate genetic counselling is essential.

Bilateral polycystic disease in infancy and childhood is now seen to have a wider clinical range than was at first thought, and may consist of several different entities. Blyth and Ockenden described four groups-perinatal, neonatal, infantile, and juvenile. ${ }^{4}$ The perinatal group presented with huge kidneys at birth, the baby being stillborn or dying within a few weeks from renal failure. In this group $90 \%$ of the renal tubules were affected and hepatic fibrosis was minimal. The neonatal group presented in the first month with large kidneys and died in the first year of uraemia; $60 \%$ of the renal tubules were affected and hepatic fibrosis was minimal. The infantile group presented at 3-6 months with large kidneys and hepatosplenomegaly, dying in childhood with systemic and portal hypertension and progressive renal failure; a quarter of the renal tubules were affected and there was considerable hepatic fibrosis. Finally, the juvenile group developed the disease in childhood, with hepatosplenomegaly and progressive portal hypertension, and most survived into their teens; here less than $10 \%$ of the renal tubules were affected, but gross hepatic fibrosis was present. Family studies suggested but did not prove that all four groups represented different autosomal recessive conditions, though other workers have considered that the difference between the groups may reflect differences in the natural history of the disorder rather than separate entities. ${ }^{5}$ Congenital hepatic fibrosis, however, is probably a separate entity. ${ }^{56}$

Blyth and Ockenden also reported for the first time cases of the adult type of polycystic kidney disease presenting in childhood. Dominant inheritance was clear in two of the patients. Since then several other probable or possible cases have come to light. ${ }^{7-10}$ Recently Shokeir has described a further six examples. ${ }^{11}$ The six infants were unrelated, and at diagnosis one was a stillborn fetus, one a liveborn baby immediately after birth, and one a neonate of 3 weeks, and three were infants ranging from $2 \frac{1}{2}$ to 4 months. The five liveborn babies died hours to weeks after the diagnosis. Other cases of the adult type were then found in the families, and in four cases parents turned out to have the disease. The disorder was transmitted as an autosomal dominant. The diagnosis in parents or other members of the family, however, was made only because the suspicion of "adult" polycystic disease in the offspring led to a specific search for it.

The adult type may be recognised in infancy during radiological investigation or on gross pathological examination: the intravenous urogram characteristically shows stretching and distortion of the caliceal system, while pathological examination shows cysts, from microscopic to gross, in both cortex and medulla with intervening areas containing normal renal tissue. In the infantile type, on the other hand, the cysts (which correspond to the type I of Osathanondh and Potter ${ }^{12}$ ) are fusiform and arranged radially throughout the kidney fairly uniformly, and this may be recognised radiologically and on gross pathological examination. Histological examination of the liver may help to differentiate the infantile from the adult type, since the characteristic intrahepatic biliary malformations are always present in the portal tracts of those with the infantile type. When there is a strong suspicion of the adult type of polycystic disease in infancy the parents and other members of the family should be investigated even if there is no history of the disease. Genetic predictions should not be based on the autosomal recessive inheritance characteristic of infantile polycystic kidney disease until autosomal dominant inheritance of "adult" polycystic kidney disease has been excluded. 